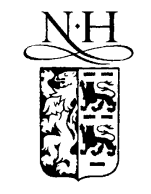

ELSEVIER

\title{
Glass foams: formation, transport properties, and heat, mass, and radiation transfer
}

\author{
Andrei G. Fedorov ${ }^{\mathrm{a}, *}$, Laurent Pilon ${ }^{\mathrm{b}}$ \\ ${ }^{a}$ G. W. Woodruff School of Mechanical Engineering, Georgia Institute of Technology, Atlanta, GA 30332-0405, USA \\ ${ }^{\mathrm{b}}$ Heat Transfer Laboratory, School of Mechanical Engineering, Purdue University, West Lafayette, IN 47907, USA
}

Received 9 October 2001; received in revised form 15 February 2002

\begin{abstract}
Energy efficiency, environmental impact, and quality of the final product in glass manufacturing depend, to a large extent, on foams formed on the surface of the molten glass and of the batch due to entrapment of gas bubbles generated by the batch fusion and refining chemical reactions during the melting process. Hence, understanding the mechanisms of foam formation as well as development of theoretical models for thermophysical and transport properties and heat, mass, and radiation transfer in glass foams are not only a problem of significant fundamental interest but also of tremendous practical impact. In this paper, the review of the current state-of-the-art in our understanding of glass foams is provided, including some of our recent results in modeling the dynamics of the foam growth and its steadystate thickness, prediction of gas diffusion through glass foams, and thermal radiative properties of glass foams. In addition, the new results on simulation of combined conduction and radiation heat transfer in glass foams and radiative transfer in primary (batch) foams are presented and discussed in some detail. The paper also presents practical means available for reducing foaming in glass melting and concludes with the discussion of unresolved problems and summary of the directions for the future work in the area.
\end{abstract}

(c) 2002 Elsevier Science B.V. All rights reserved.

PACS: 44.40.+a; 47.55.Dz; 82.70.Rr; 83.70.Hq

\section{Introduction}

The glass manufacturing industry provides products that are critical to a wide range of industries from such low-tech items as glass containers and decorations to such high-tech items as

\footnotetext{
${ }^{*}$ Corresponding author. Tel.: +1-404 385 1356; fax: +1-404 8948496.

E-mail address: andrei.fedorov@me.gatech.edu (A.G. Fedorov).
}

fiber optics and optoelectronic devices. According to a Gas Research Institute report (1995), the US glass industry also represents a major source of natural gas demand - approximately 5.5 billions of cubic meters per year, of which about $90 \%$ is used for glass melting and the rest is used for downstream processing. The scope and importance of the glass manufacturing industry are underscored by the following statistical facts: it employs about 150,000 people; approximately 22 million tons of glass are melted each year; and it has around $\$ 23,215,000,000$ market value in the US. 


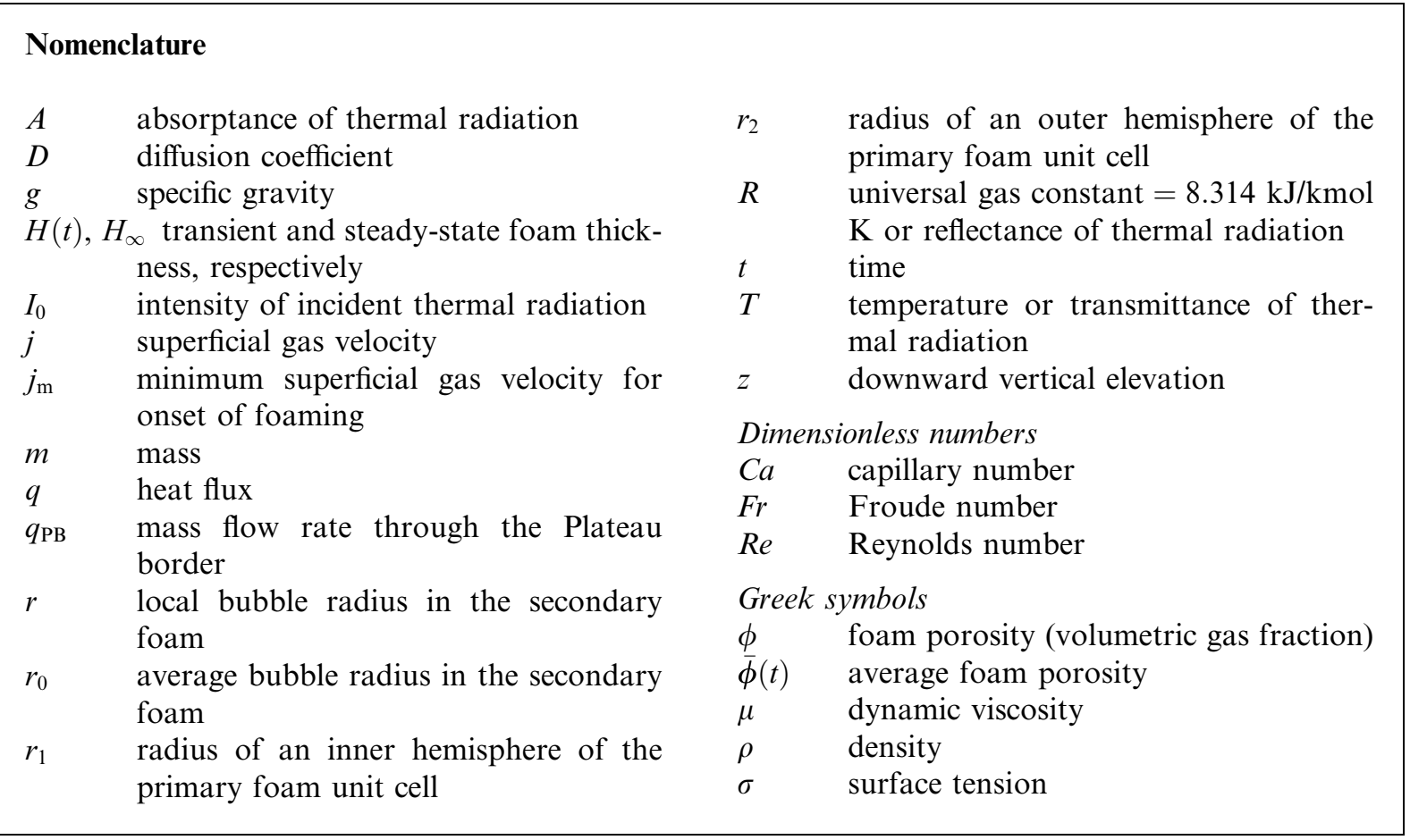

During the past two decades, business competition and economic problems have forced glass manufacturers to increase productivity and product quality. In addition, combustion-generated pollutant emissions may be an equal or even more important challenge faced by glass companies. An empirical, experience-based approach to design and operation of glass melting furnaces fails to meet these challenges, forcing glass industry to explore the possibilities of using physics-based models of the melting process and advanced computer simulation tools for optimizing existing processes and designing the next generation of glass melters. The physico-chemical processes taking place during glass melting are extremely complex with the heat and mass transfer issues being of key importance. A schematic of the glass melting furnace is shown in Fig. 1. It indicates the heat input by convection and radiation from the combustion space $\left(q_{\text {top }}\right)$ to the top of the piles of raw materials (batch) and from the hot molten glass $\left(q_{\text {bot }}\right)$ to the bottom, submerged part of the batch. In an electrically boosted glass tank, an additional energy for melting batch is supplied by
Joule heating of the glass due to the passage of electrical current between electrodes inserted in the bath. In addition, heat transfer from the combustion space to the glass surface drives glass circulation in a glass melting tank to achieve complete melting of raw materials, homogenization of the melt, and to remove all bubbles from the melt prior to using it for the final processing. The batch enters the furnace through the ports and is partially submerged into the molten glass. It is pushed in and spreads over the surface of the glass by the advection currents in the glass melt while being heated, fused, and melted. The fusion of raw materials in the batch generates a large number of gas bubbles, and a fraction of these bubbles is entrapped within the batch and in the primary melt formed on top of batch logs to produce the primary foam (Fig. 2(a)) [1].

Bubbles generated at the bottom of the batch layer are trapped in the melt and carried by the advection currents present in the glass bath. Refining agents are usually added to the batch in order to facilitate bubble growth and removal 


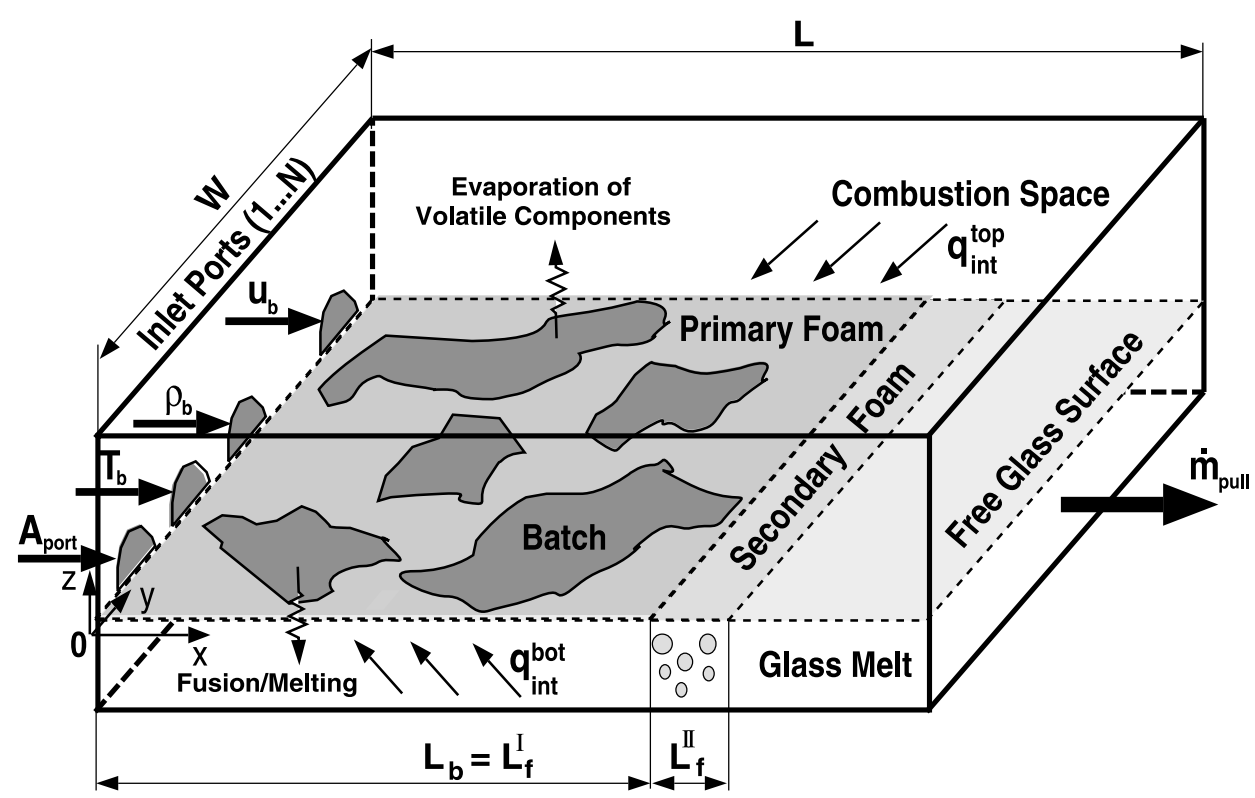

Fig. 1. Schematic of a glass melting furnace.

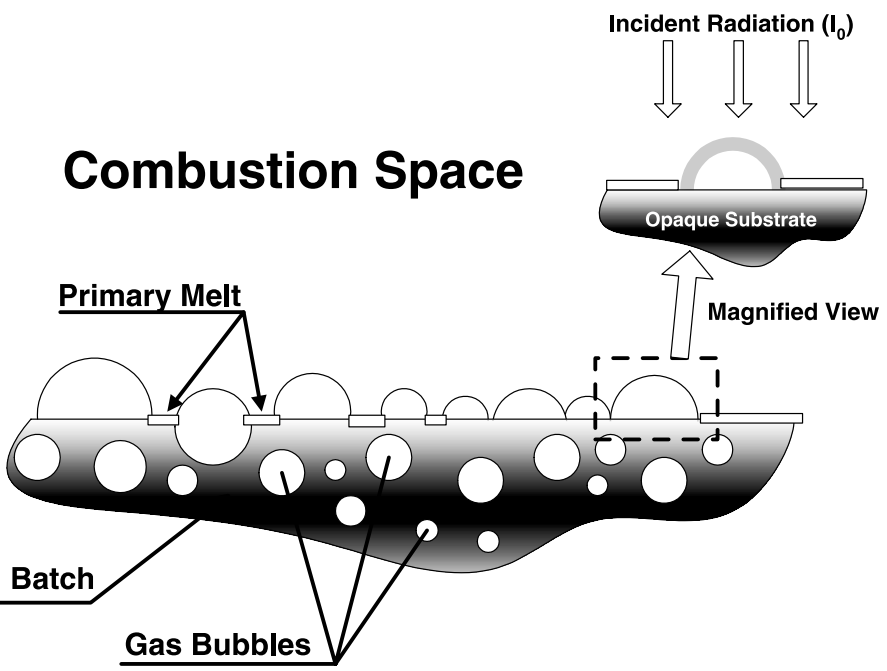

(a)

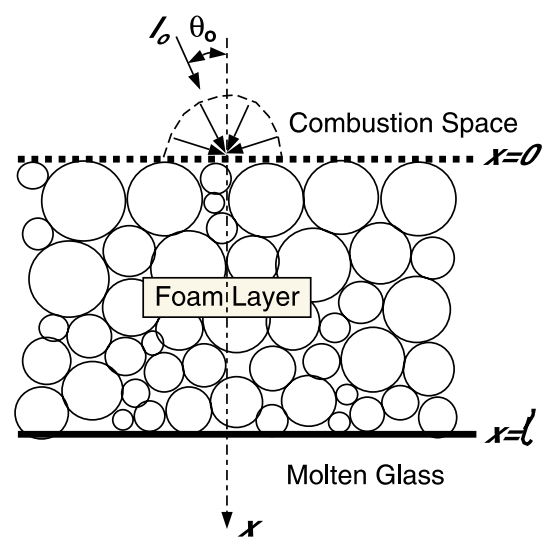

(b)

Fig. 2. (a) A layer of the primary foam formed on top of batch logs with a magnified view showing an idealized unit cell used for analysis of radiative transfer, and (b) an idealized layer of the secondary glass foam.

from the glass melt [2-4]. These agents mediate equilibrium redox reactions that produce or consume gases depending on the local conditions in the glass. In particular, in high temperature regions, the refining reactions generate fining gases that either form new bubbles through nucleation or dissolve in the glass melt and eventually diffuse into existing gas bubbles. The buoyancy force enables sufficiently large bubbles to rise to the free surface of the glass melt, where they may build up 
and lead to formation of the so-called secondary foams (Fig. 2(b)) [1].

\section{Motivation}

There exists a number of important fundamental and practical reasons for studying formation, physicochemical and transport properties, and heat, mass, and radiation transfer in glass foams. These are:

- Energy efficiency: As indicated earlier, fusion and melting of the batch raw materials require a significant amount of energy, which is predominantly supplied by thermal radiation from the hot combustion products and heated furnace refractories above the melt [5]. According to indirect measurements and estimations by Trier [6], the resistance to radiative heating due to the presence of foams is significant, and it could lead to a decrease by as much as a $60 \%$ in radiative fluxes to the batch and glass melt. This results in significant reduction of the energy efficiency of the furnace and an increased fuel consumption [2,7] required to reach a sufficient glass melt temperature. Moreover, the batch expansion or primary foaming is held responsible for decreasing the batch melting rate [8].

- Glass quality: An attenuation of heat transfer from the combustion space to the glass melt reduces the glass bath temperature and, hence, limits the rate of refining reactions, thereby increasing the number of bubbles contained in the final product, which is detrimental to its quality [7].

- Productivity: Presence of the foam leads to an increase in the residence time for the glass melt in the furnace which is required to attain needed quality characteristics/indices of the glass. Moreover, it has been observed [1,9] that an increase in the pull rate favors foaming, thereby limiting the maximum pull rate allowed to sustain high efficiency operation and satisfactory quality of the glass prior to its processing.

- Pollutant emissions: Reflection or back-scattering of thermal radiation by foams results in a considerable increase in combustion-generated $\mathrm{NO}_{x}$ pollution, owing to an increase in the refractories temperature by several hundreds of degrees Celsius. In addition, mass diffusion in glass foams regulates the chemical species transport across the molten glass/combustion space interface and, thus, is very important to predicting the chemical composition and quality of the glass. Gas diffusion through the glass foam is a thermally activated process, and the effective mass diffusion coefficient for major gas species $\left(\mathrm{O}_{2}, \mathrm{SO}_{2}, \mathrm{CO}_{2}\right.$, and others) depends exponentially on the temperature.

- Furnace integrity: It has been observed that presence of the foam enhances the refractories attack at the metal-glass line and also promotes the wear of the crown due to an increased process temperature [10].

- New melting technologies: Attracted by apparent environmental and economic advantages, new industrial practices gravitate towards the use of oxy-fuel burners and significant amounts of the recycled cullet in the batch raw materials; however, both of these measures have been shown to favor the foam formation with all above mentioned negative effects $[1,11]$. The oxygen firing burners produce larger fraction of the water vapor in the combustion products that is believed to diffuse into the glass melt, thereby influencing important physico-chemical properties of the glass melt such as viscosity, sulfate chemistry, the redox state of the glass melt, and the color of the glass [2]. On the other hand, melting of the cullet occurs at much lower temperatures resulting in a viscous liquid layer that covers the batch logs and prevents the gas bubbles from escaping freely to the combustion space and, thus, enhancing the primary foam formation [11].

These facts underscore the critical importance of detailed understanding of formation, stability, and heat and mass transfer in the batch and glass foams not only for improving the efficiency and environmental safety of the glass melting process but also to the quality of the final glass products. Clearly, developing a fundamental understanding of mechanisms of foam formation and the ability 
to predict the effective transport properties and, in turn, heat, mass, and radiation transfer in glass foams is one of the keys to designing the energy efficient and environmentally friendly glass melting technology.

\section{Background}

\subsection{Primary foam or batch expansion}

Prior to melting the batch goes through the heating and fusion stages, involving exo- and endothermic solid-state reactions between various batch components [12]. As the temperature increases beyond $800{ }^{\circ} \mathrm{C}$, liquid phases (also called primary melt) begin to appear. Formation of the primary melt is accompanied by generation of significant amounts of carbon dioxide through decomposition of carbonates present in the batch. A part of the released $\mathrm{CO}_{2}$ is trapped in the viscous liquid phase, whereas the remaining gas transpires through the open pores in the batch [12]. As the melting process proceeds, the melt fraction and its connectedness increase, the open pores become filled by the molten glass, thereby trapping the gas bubbles within the batch and leading to batch expansion [12]. Eventually, as the number of entrapped bubbles increases, they start coalescing within the batch and form open channels through which the entrapped gases escape to the atmosphere. The presence of trapped gases within the batch lowers its effective thermal conductivity and, in turn, reduces the melting rate [8].

Refining reactions also take place in the high temperature (beyond $1100{ }^{\circ} \mathrm{C}$ ) regions of the batch. Thus, if the batch reaches temperatures larger than $1100{ }^{\circ} \mathrm{C}$ it undergoes two consecutive expansions: the first one is due to $\mathrm{CO}_{2}$ release, and the second is due to generation of refining gases [12]. Note that in the carbon-containing batch, carbon dioxide $\mathrm{CO}_{2}$ partly reacts with carbon-containing components to generate carbon monoxide $\mathrm{CO}$ at the temperatures above 750 ${ }^{\circ} \mathrm{C}$. The latter becomes entrapped in the batch and can react with sodium sulfate (refining agent) to form $\mathrm{SO}_{2}$ gas causing further batch expansion [13].
Unlike the foams formed on the free surface of the molten glass that possess a structure of multiple layers of spherical or polyhedral bubbles separated by the liquid lamellae, the batch foam formed on top of the batch logs is a single layer of closely packed, and only partially immerged individual gas bubbles whose unit cell can be realistically represented by a thin hemispherical shell placed on the radiatively opaque surface of the batch (Fig. 2(a)).

\subsection{Secondary foams}

Secondary foams (Fig. 2(b)) are formed in glass melting furnaces due to the gas bubbles, which are released during the refining chemical reactions in the glass melt and entrapped at the glass free surface $[1,9]$. Refining agents, which are involved in equilibrium redox reactions producing or consuming gases, are usually added to the batch to remove undesirable bubbles from the glass melt [14-16]. As the refining agents are carried by the flow, they encounter high temperature regions (beyond $1400{ }^{\circ} \mathrm{C}$ ) in the glass melt where refining reactions take place and gas is being generated. In addition, some of the bubbles generated as a result of batch fusion and melting reactions and trapped under the batch are carried by the convection currents in the glass bath and eventually rise to the glass free surface, also producing the secondary foam [1].

In general, three types of refining agents are commonly used [1]:

1. Variable-valence metal oxides which release only oxygen, e.g., the antimony oxides $\left(\mathrm{Sb}_{2} \mathrm{O}_{5} /\right.$ $\left.\mathrm{Sb}_{2} \mathrm{O}_{3}\right)$, the arsenic oxides $\left(\mathrm{As}_{2} \mathrm{O}_{5} / \mathrm{As}_{2} \mathrm{O}_{3}\right)$, the cesium oxides $\left(\mathrm{CeO}_{3} / \mathrm{CeO}_{2}\right)[14,15]$.

2. Sulphates and sulphites which release a mixture of sulfure oxide $\left(\mathrm{SO}_{2}\right)$ and oxygen [2]. For example, sodium sulfate $\mathrm{Na}_{2} \mathrm{SO}_{4}$ is commonly used as a refining agent at high temperatures (around $1800{ }^{\circ} \mathrm{C}$ ), but it also accelerates the primary melt formation if introduced into the batch in suitable proportions [17]. However, a satisfactory explanation of beneficial effects associated with using sulphates as refining agents is not yet available. 
3. Chlorides, bromides, and iodines which evaporate at elevated temperatures rather than participate in refining reactions [18].

The secondary foam layer of varying thickness (usually only a few centimeters) covers about onethird of the molten glass surface, mostly in the part of the furnace where batch is still present (Fig. 1). Being a highly porous and inhomogeneous colloidal system of gas bubbles separated by thin glass lamellae, foam essentially acts as a collection of scatterers imbedded into a semitransparent material, thereby providing resistance to thermal radiation heating of the batch and of the glass melt $[19,20]$.

\subsection{Foam structure}

Glass foams, at least secondary ones, belong to so-called pneumatic foams, which are produced by a continuous stream of gas bubbles rising to the surface of a foaming liquid. Pneumatic foams consist of an ensemble of bubbles of different sizes, and the size distribution function varies across the foam layer. The bubbles at the bottom of the foam layer are usually spherical in shape, and their size distribution is primarily defined by the injection system [21]. The bubbles at the top of the foam layer are usually polyhedral, and their geometry typically obeys the two universal rules called the Plateau's laws [22]: (1) three and only three films or lamellae, called Plateau borders, meet at an edge of a polyhedral bubble at an angle of $120^{\circ}$, and (2) four and only four edges, called Plateau border channels, meet at a point at an angle of $109^{\circ}$. The dodecahedron nearly satisfies the Plateau's laws and, thus, commonly used as an idealized model for polyhedral bubbles in the foam.

Laimbock [1] observed polyhedral bubbles in the glass foam generated by bubbling gas through the molten glass in the laboratory crucible. However, such a foam morphology may not be characteristic to an actual glass melting process in the industrial furnaces, where bubbles are generated by chemical reactions and have been observed to be much smaller than those generated by gas injection in the laboratory system [23]. This is supported by the analysis of the glass foam samples collected in actual melting furnaces [24] for which the average bubble radius is of the order of 0.5 $\mathrm{mm}$, whereas the laboratory glass foams generated by bubbling gas through the melt [1] have an average void radius between 15 and $20 \mathrm{~mm}$.

\subsection{Mechanisms of formation}

A number of intimately interacting physical phenomena govern the dynamics of the foam formation and its steady-state thickness. It includes (1) bubble built-up in the foam due to the bubble influx from the bottom of the foam layer; (2) drainage of the lamella liquid from the Plateau borders induced by the capillary suction forces and opposed by the disjoining pressure forces; (3) gravity induced drainage of the liquid from the foam through the Plateau border channels; (4) abrupt liquid discharge from the foam due to the rupture of the lamellae and coalescence of adjacent bubbles; (5) drainage of the liquid from the foam due to so-called disproportionation or an Ostwald ripening effect owing to interbubble gas diffusion from smaller bubbles (higher pressure) to larger bubbles (lower pressure) [21,22,25].

Different mechanisms dominate dynamics of the foam formation during different stages of the process: initially, foam growth is primarily defined by the balance between the bubble built-up in the foam due to the gas influx (mechanism 1) and the liquid drainage from Plateau borders and Plateau border channels (mechanisms 2 and 3); however, in the later stage of the process, when the foam is sufficiently drained and the lamellae separating bubbles in the foam become thin, the bubble coalescence (mechanism 4) and interbubble gas diffusion (mechanism 5) tend to become dominant at least at the top of the foam.

\section{Current state of knowledge}

A tremendous cost and often simply impossibility associated with empirical on-the-plant optimization of the thermal design and operation of glass melting furnaces provided a strong boost to fundamental research in transport and chemical processes underlying glass melting, their mathe- 
matical modeling and laboratory scale experimentation. Although heat, mass, and radiation transport in the combustion space (see review by Viskanta and Menguc [26]) and in the glass melt $[5,27]$ has been studied quite extensively, heat and mass transfer in glass foams has been essentially neglected.

Although an importance of foams to glass melting technology has been realized long time ago [6], there have not been significant advances in our understanding of glass foams including mechanisms of their formation, effective transport properties, and heat, mass, and radiation transfer in foams. Only very recently, Laimbock [1] presented an extensive experimental study concerned primarily with dynamics of foam formation and mass transfer across the glass foams. In parallel, in a series of papers [19,20,28-34], we have attempted to gain theoretical understanding and develop experimentally validated mathematical models of the key transport phenomena in glass foams.

\subsection{Dynamics of glass foam formation}

Over the years, a number of theoretical models of various levels of sophistication have been proposed to predict the dynamics of the pneumatic foams, including foams formed in molten glass (see $[21,22,25]$ for relevant literature citations). More sophisticated models use the population balance theory and account for drainage, coalescence, and interbubble gas diffusion; however, these models have only been successfully applied to predicting the dynamics of the decay, not growth of the foams (see [22] and references cited therein). In addition, the more complex and sophisticated the model becomes, the larger number of input parameters, which are often not readily available, is required to perform simulations. The extreme operating temperatures and the process complexity that are characteristic to glass manufacturing provide serious fundamental limitations to measuring some of the key parameters (e.g., solubility and diffusivity of the gases in the glass melt), which are needed for more sophisticated models of the foam growth.

To this end, we have devised a simple yet realistic model of the foam dynamics [30], whose predictions are supported by the available experimental data. Three distinctly different regimes in the transient growth of the foam have been identified as a function of the superficial gas velocity (Fig. 3): (i) at low superficial gas velocities, the foam thickness increases linearly with time and quickly reaches a steady-state, (ii) at intermediate superficial gas velocities, the foam thickness exhibits large transient oscillations and never reaches a steady-state, and (iii) at large superficial gas velocities, the foam thickness initially increases linearly with time and then foam suddenly collapses into a steady-state froth. The model assumes that the entire time interval during which the foam grows from the zero thickness to its steady-state thickness can be divided into two parts: first is a very small time interval in the beginning of the foam formation when the foam built-up due to bubble influx and the foam decay due to primarily drainage from the Plateau borders and Plateau border channels are comparable in magnitude; and the second part is when the foam is sufficiently drained and its dynamics is dominated by the balance between the bubble built-up and interbubble gas diffusion and bubble coalescence and collapse near the top of the foam. In both cases, the mass conservation dictates that the transient thickness of the foam is linearly proportional to the bubble injection superficial velocity and inversely proportional to the thickness averaged porosity of the foam, i.e., $H(t)=j t / \bar{\phi}(t)$ [30]. However, during the first, drainage dominated part of the process, the average porosity of the foam layer changes significantly with time and it is obtained by solving of a so-called drainage equation [22]. During the second, coalescence and rapture dominated stage of the process, the foam is already well drained at the top and consists of primarily polyhedral bubbles; thus, coalescence and rapture do not affect the geometric structure (porosity) of the foam but only change the average size of bubbles. Hence, in the second stage of the process, the average porosity $\bar{\phi}(t)$ can be essentially treated as independent of time and equal to $\left(\phi_{\text {top }}+\phi_{\text {bottom }}\right) / 2$, if the linear porosity profile is assumed or equal to $\left(2 \phi_{\text {top }}+\phi_{\text {bottom }}\right) / 3$, if the exponential porosity profile is assumed. Here, $\phi_{\text {top }}$ denotes the porosity at the top of the foam layer 

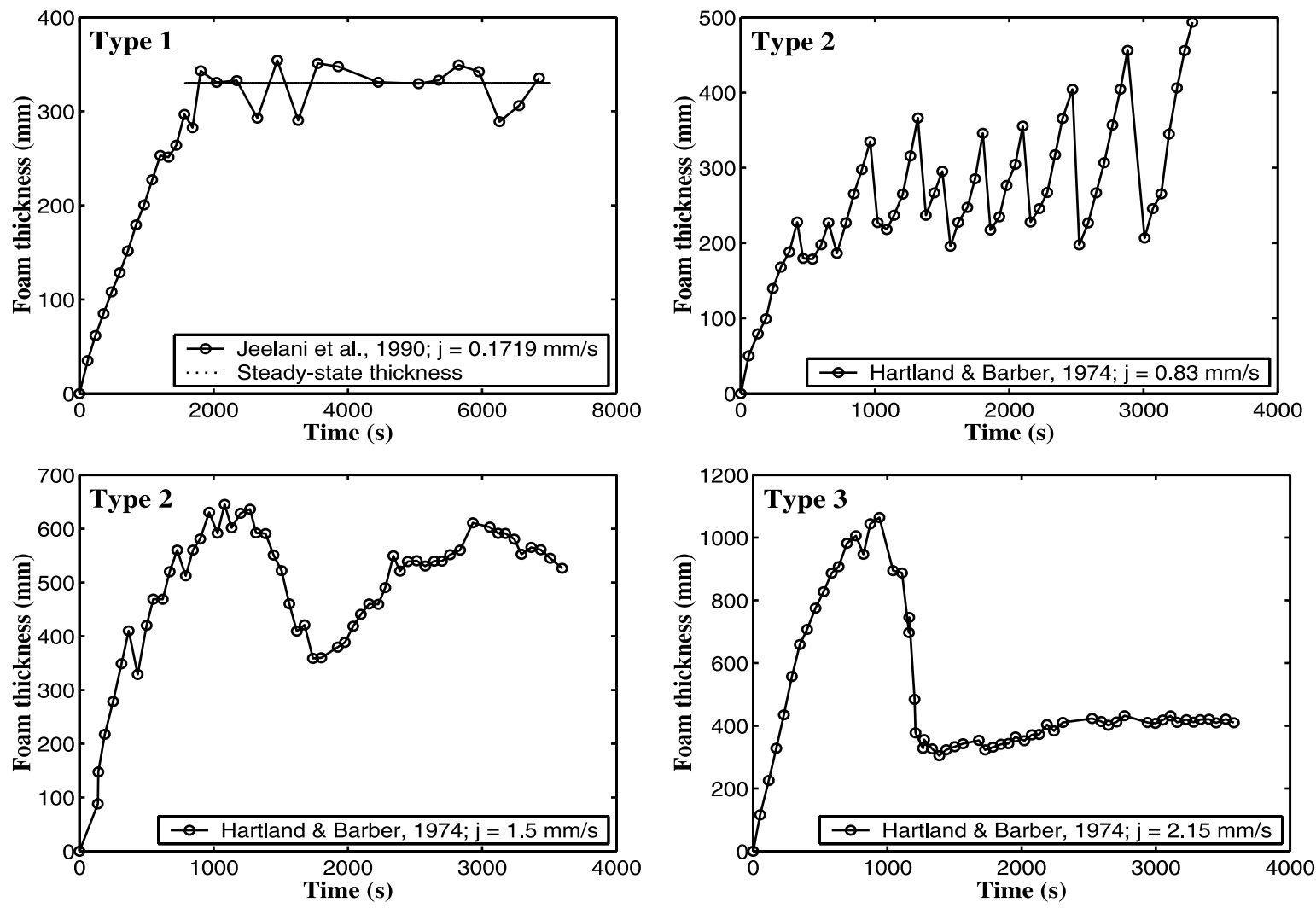

Fig. 3. Dynamic regimes of transient evolution of the foam thickness $[30,35,36]$.

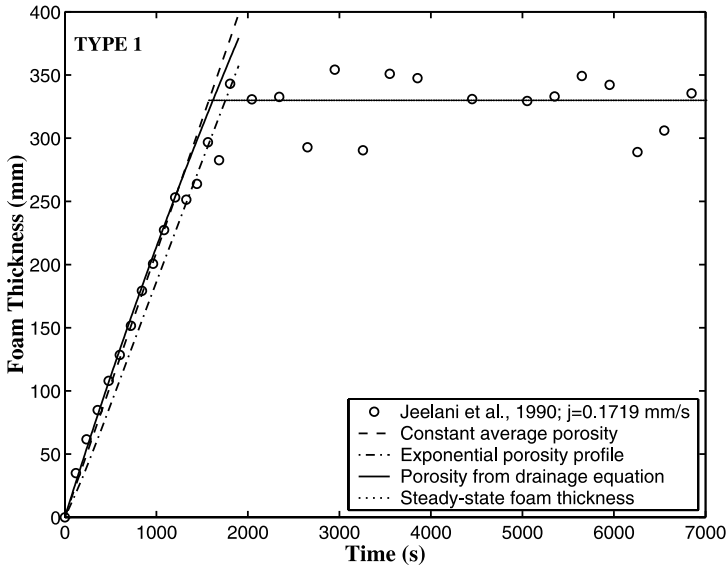

Fig. 4. Transient foam thickness: Comparison of the model predictions with experimental data for nitrogen in an aqueous solution of $10 \%$ glycerine and $80 \mathrm{mg} / \mathrm{l}$ of marlophen- 89 [36]. (approximately 0.98 for closely packed polyhedral bubbles), and $\phi_{\text {bottom }}$ is the porosity at the bottom of the foam layer (equal to 0.74 for closely packed spherical bubbles). Fig. 4 compares the predictions of the model against the experimental data [30]. Clearly, a very good agreement between the theoretical predictions and experimental results is obtained, thereby providing a confidence in the proposed physical description of the process as well as in the developed theoretical model.

\subsection{Steady-state thickness of glass foams}

As shown later in this review, the steady-state thickness of the glass foam is the most important parameter defining its effective transport properties and the rate of heat, mass, and radiation transfer across the foam. In addition to glass melting, the problem of predicting the steady-state 
thickness of the foam has been of great interest because of the broad range of applications including steel manufacturing, oil, petroleum, and pharmaceutical industries. For this reason, a number of models have been proposed to correlate the steady-state foam thickness to the main operating parameters (a bubbling velocity, a bubble size distribution, and others) and thermophysical properties of the injected gas and of the foaming solution. As in the case of modeling the transient behavior of foams, the models developed could be broadly divided into the two groups [29]: (1) simple, largely empirical correlations that lack generality and are valid for only very narrow range of operating conditions; and (2) very sophisticated models, which are rather general, but require a large number of input parameters that are usually not available or difficult to obtain from the experiment. The latter models have also received very limited validation against the experimental data and, thus, are met with skepticism by the practitioners.

Only recently, Pilon et al. [29] have developed a model based on first principles that shows a remarkable capability in matching the experimental data for a number of very different bubbling gases (air, nitrogen, argon) and foaming solutions (including molten glass) over the wide range of experimental conditions (superficial bubble velocity $j$ variation is between 0 and $40 \mathrm{~mm} / \mathrm{s}$, the mean bubble radius $r_{0}$ variation is between 0.7 and 20 $\mathrm{mm}$, viscosity $\mu$ variation is between 46 and 12,100 $\mathrm{mPas}$, density $\rho$ variation is between 1200 and $3000 \mathrm{~kg} / \mathrm{m}^{3}$, and surface tension $\sigma$ variation is between 69.5 and $478 \mathrm{mN} / \mathrm{m}$ ). To develop the model, we performed a dimensional scaling analysis of conservation equations governing the dynamics of the transient foam growth [22] and identified only two dimensionless numbers as necessary to describe the stability of the pneumatic foams. These numbers are

$\Pi_{1}=R e / F r \quad$ and $\quad \Pi_{2}=C a \times\left(H_{\infty} / r_{0}\right)$

where $R e$ is the Reynolds number $\left[=\rho\left(j-j_{\mathrm{m}}\right) r_{0} /\right.$ $\mu], F r$ is the Froude number $\left[=\left(j-j_{\mathrm{m}}\right)^{2} / g r_{0}\right], C a$ is the capillary number $\left[=\mu\left(j-j_{\mathrm{m}}\right) / \sigma\right], H_{\infty}$ is the steady-state foam thickness, $r_{0}$ is the mean bubble radius, and $j_{\mathrm{m}}$ is the minimum superficial bubble velocity required for onset of foaming. It should be emphasized that there is a minimum superficial gas velocity that is required to initiate foaming, and this fact has been confirmed experimentally for glass foams by Laimbock [1]. The power-law type relationship $\left(\Pi_{2}=K \times \Pi_{1}^{n}\right)$ has been proposed based on the analysis of the physical mechanisms responsible for the foam stability under the steadystate conditions, and the empirical parameters $K=2905$ and $n=-1.8$ have been obtained using an extensive experimental data collected from the literature (see Fig. 5(a)). The resulting model, expressed as

$H_{\infty} / r_{0}=(2905 / \mathrm{Ca}) \times(\mathrm{Fr} / \mathrm{Re})^{1.8}$

has been thoroughly validated by comparing its predictions of the steady-state foam thickness with the experimental data as shown in Fig. 5(b). Clearly, a remarkably good agreement is observed given the wide range and uncertainty of the experimental data used for comparison. It should be noted that this model was developed and validated for onedimensional laboratory foams generated in small vertical containers without considering the effects of lateral motion (spreading) of the foam that is typical to glass melting. Thus, the next logical step would be to extend the model by accounting for the rheological properties of foams and formulating transport equations that govern foam behavior in two- or three-dimensional settings.

\subsection{Effect of the furnace atmosphere and bubble gas composition on foam stability}

It has been observed that foaming of glass increases with the use of pure oxygen burners [1]. Similarly to metallurgical slag foams [37], the type of gaseous fuel used and the luminosity of the flame are also expected to have a marked impact on the production and stability of glass foams. The physical reasons causing such effects are not clearly understood, although the flame temperature and pressure as well as a chemical makeup of combustion products (especially, water vapor mass fraction) are thought to be the major factors [37].

The atmosphere on the top of the foam layer contains from $40 \%$ to $60 \%$ of water in oxygen-fuel fired glass melting furnaces, whereas it contains 

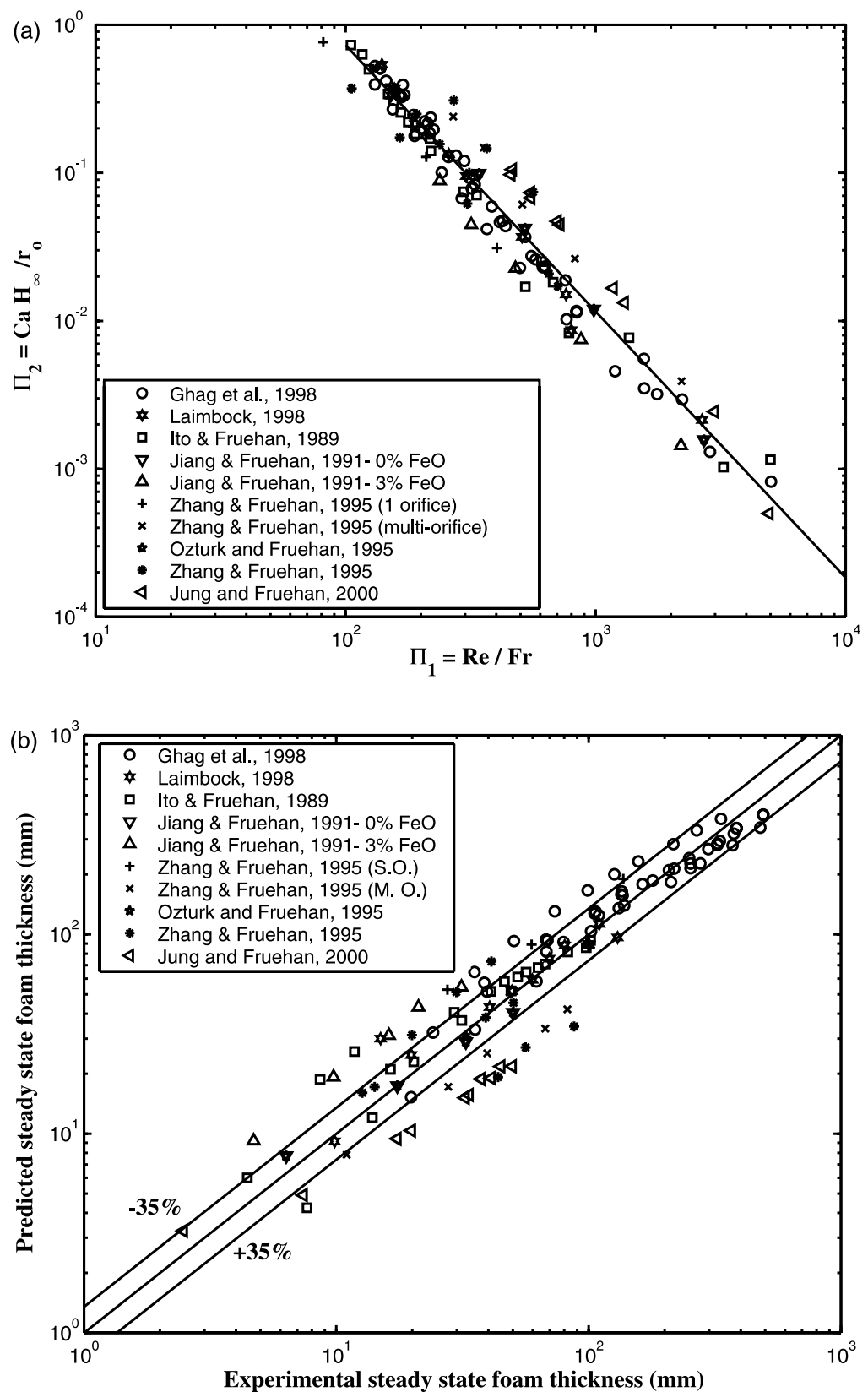

Fig. 5. Steady-state foam thickness: (a) correlation between the key dimensionless numbers; (b) comparison between the predictions and experimental data.

only $12-18 \%$ in air-fuel fired furnaces and $1-2 \%$ in the ambient air. Cable et al. [38] studied the foaming behavior of binary silicate melts and concluded that the atmosphere composition had a significant effect on the foam. This group and other researchers experimentally observed that (i) foaming temperature was lower in a dry atmosphere, (ii) no foam was observed in the pure nitrogen atmosphere, (iii) glass foams are more stable in the pure oxygen atmosphere, confirming 
visual observations by Laimbock [1], and (iv) strongly reducing furnace atmosphere destabilizes the foam significantly. The effect of water vapor on the foam stability appears to depend on the foaming agent. It has been experimentally observed that an increase in the partial pressure of water in the furnace atmosphere stabilizes the foam made from binary silicate [38] and soda-lime silicate [1] glasses containing sulfate. However, water vapor destabilizes the foam obtained from binary silicate [38] and soda lime silicate [9] containing carbonate instead of sulfate. Therefore, the interactions of sulfate with water dissolved into the melt seem to be essential to foam formation and have been extensively discussed by Beerkens and Laimbock $[1,11,39]$.

It is interesting to note that an independent study by Parikh [40] of the effect of the atmosphere composition on the surface tension of soda-lime silicate glass indicates the same trends. In particular, non-polar gases such as dry air, nitrogen, helium and hydrogen have no effect on the surface tension and, thus, on the foam stability. On the other hand, polar gases such as sulfure dioxide $\left(\mathrm{SO}_{2}\right)$, ammonia $\left(\mathrm{NH}_{3}\right)$, hydrogen chloride $(\mathrm{HCl})$, and water vapor $\left(\mathrm{H}_{2} \mathrm{O}\right)$ reduce the surface tension of glass by different amounts [40]. Among all the polar gases cited, water vapor has the largest dipole moment and, in turn, has the most significant effect on the surface tension. In fact, Parikh [40] showed that the surface tension decreases as the square root of the partial pressure of water vapor in the atmosphere. He attributed this observation to the adsorption of water vapor at the glass/ atmosphere interface and to saturation of the water vapor in glass caused by the presence of sodium ions $\mathrm{Na}^{+}$at the interface. In addition, when water vapor is present in the atmosphere, a monolayer of $\mathrm{OH}^{-}$ions is formed in the top surface of the foam, thereby also causing the surface tension to decrease [41]. Above mentioned experimental facts suggest that an increased foaming and the higher thickness of the glass foams observed in the furnaces outfitted with the pure oxygen burners are primarily due to an increase in the partial pressure of water vapor in the furnace atmosphere resulting in a reduced surface tension of the glass which, in turn, stabilizes the foam layer.
The effect of water vapor on chemistry of sulfate-based refining agents should be also explored to explain the experimental observation of an increased foaming in the oxi-fuel furnaces. Indeed, Beerkens et al. [13] found experimentally that water vapor dissolved in oxidized melt enhances fining reactions involving sodium sulfate and reduces the foaming temperature of soda-lime silicate glasses. Moreover, water vapor infiltration increases in the presence of the foam, thereby further favoring refining reactions in the melt and, in turn, foam formation [13]. The same phenomena are expected to occur when variable-valence metal oxides (e.g., the antimony oxide) are used as refining agents instead of sodium sulfate [1].

\subsection{Effective transport properties of glass foams}

Pilon et al. [28] have investigated mass diffusion in glass foams, as it regulates the species transport across the molten glass/combustion space interface and thus is very important to predicting the gas transport in the glass melt. Using similarity between heat and mass transfer processes, Varady and Fedorov [33] also developed the model for the effective thermal conductivity of glass foams based on the previous work on mass diffusion in glass foams [28]. The models for effective mass and heat diffusion coefficients have been developed using an equivalent resistance network for the cubic unit cell of the foam (Fig. 6). In general, the foam structure (porosity $\phi$ and void/unit cell morphology), the thermal solubility parameter i.e., the product of the solubility $(S)$, foam temperature $(T)$, and gas constant $(R)$, and the diffusion coefficient of a given gas in the condensed liquid phase $\left(D_{\mathrm{c}}\right)$ are the main parameters affecting the effective diffusion coefficient [28]. In most practical cases, the voids (bubbles) in the foam do not introduce a significant resistance to mass transfer as compared to the liquid lamellae, and the effective diffusion coefficient of the entire foam layer can be expressed as

$D_{\text {eff }}=D_{\text {co }} \mathrm{e}^{-E_{\mathrm{c} / \mathrm{RT}}} \times\left[1+\phi /\left(1-\phi^{1 / 3}\right)\right] / n$

where $n$ is the average number of bubble (unit cells) across the foam layer and $E_{\mathrm{c}}$ is an activation energy for mass diffusion of a given gas in the condensed (liquid) phase. 

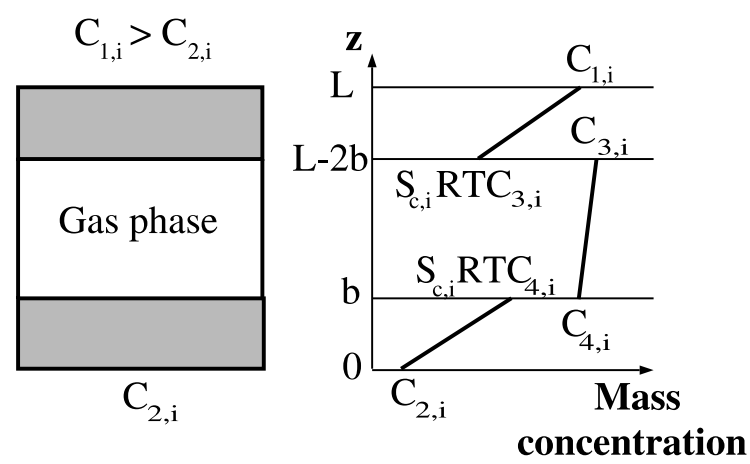

Fig. 6. Mass diffusion in isothermal glass foams: concentration profile across the foam unit cell used to compute the effective foam diffusion coefficient as a function of the foam porosity for gases with different thermal solubility parameter (the product of the solubility $S$, foam temperature $T$, and gas constant $R$ ) in the foam lamella.

Another key finding of this study, obtained theoretically and verified by comparison with experimental data, is that mass diffusion in glass foam is thermally activated process and the effective mass diffusion coefficient for major gas species $\left(\mathrm{O}_{2}, \mathrm{SO}_{2}, \mathrm{CO}_{2}\right.$, and others) depends exponentially on the temperature. This fact underscores the critical importance of detailed understanding of heat transfer (i.e., temperature distribution) in glass foams not only to improving the process efficiency and environmental safety of the glass melting process but also to the quality of the final glass products.

\subsection{Effective radiative properties of glass foams}

The effective radiative properties of the foam can be readily obtained if the foam structure (i.e., a size and morphology of the unit cell) and radiative properties of a unit cell of the foam are known [19]. Hence, one needs to understand and to quantify the absorption and scattering cross-sections as well as the scattering phase function for the unit cells that are representative to primary and secondary glass foams. The challenge is that the bubble (unit cell) morphology changes from perfectly spherical at the bottom of the secondary foam layer to polyhedral at the top of the foam layer due to the gravity induced drainage [1]. In addition, the bubble (unit cell) size and the thick- ness of the glass lamella separating bubbles in the foam could span several orders of magnitude, ranging from $0.1-3 \mathrm{~mm}$ for the bubble radius and $0.01-0.1 \mathrm{~mm}$ for the lamella thickness (spherical bubbles with the average foam porosity of 0.74 ) to $3-10 \mathrm{~mm}$ for the bubble radius and $1-10 \mu \mathrm{m}$ for the lamella thickness (polyhedral bubbles with the average foam porosity of 0.97 ). Clearly, the unit cells of the foam with different sizes and morphology have very different radiative properties, and only limited such information is currently available.

A simplified theoretical approach for predicting the effective radiative properties (absorption and scattering coefficients and the scattering phase function) of the glass foams and radiative transfer in the glass foam blanket has been recently developed by Fedorov and Viskanta $[19,20]$. Both cases of collimated and diffuse radiation incidence have been considered, and analytical expressions for the total apparent reflectance $(R)$, transmittance $(T)$, and absorptance $(A)$ of the secondary foam layer (Fig. 2(b)) have been obtained by accounting for external and internal reflecting boundaries of the layer [19]. The results of this work (see Figs. 7 and 8 for major highlights) confirm Trier's [6] indirect observations that the transmittance of thermal radiation even through a relatively thin $(2 \mathrm{~cm}$ thick) foam layer never exceeds $20 \%$ or, in other words, the foam blanket provides significant resistance to radiative heat transfer from the hot combustion products and refractories to the molten glass in the tank. We also found that the thickness of the foam layer, the behavior of the scattering/phase distribution function, bubble size and shape (spherical vs. polyhedral) distributions in the foam have major impact on the radiative characteristics of the foam. It should be also mentioned that the theoretical predictions of the total apparent radiation properties of the foam appear to be in a good agreement with the experimental data obtained at Schott Glas, Inc (Mainz, Germany). ${ }^{1}$

\footnotetext{
${ }^{1}$ This information has been disclosed to one of the authors of this paper (AGF) by the members of Schott Glas R\&D staff during his invited seminar presentation on February 8, 2001.
} 


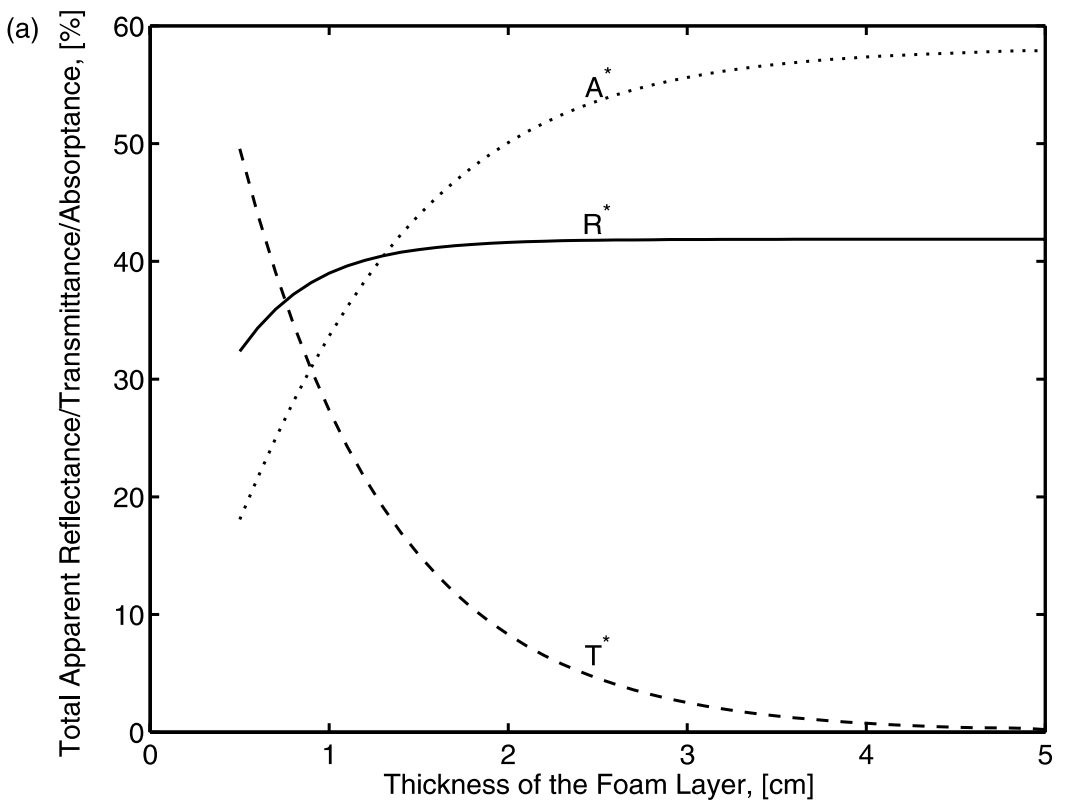

(b)

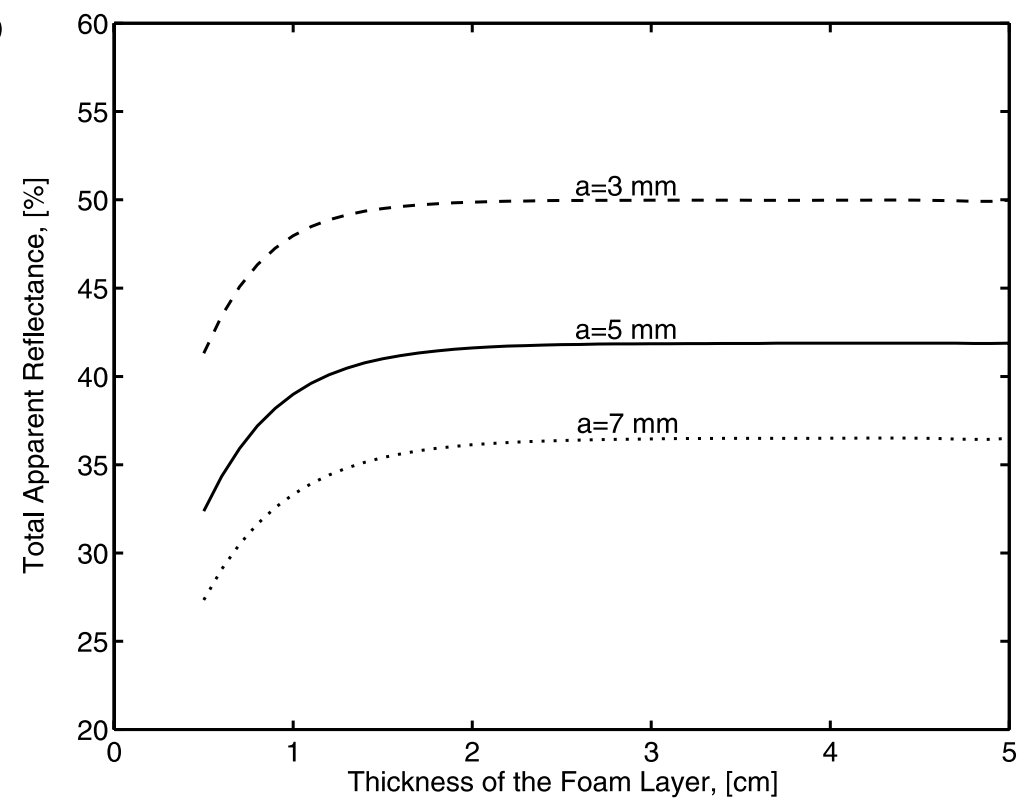

Fig. 7. (a) Radiation characteristics (reflectance $R$, transmittance $T$, and absorptance $A$ ) of the glass foam layer as a function of the foam thickness, and (b) effect of the foam bubble size on the reflectance of thermal radiation by the glass foam layer (a is the average bubble size).

Recently, we have also developed a simplified analytical model for predicting the radiative properties of the primary foams generated on top of the batch logs $[31,32]$. Specifically, by using an analytical ray tracing, we were able to obtain a closed-form, albeit approximate, analytical expressions for the total apparent absorptance, transmittance, and reflectance of a thin hemispherical 

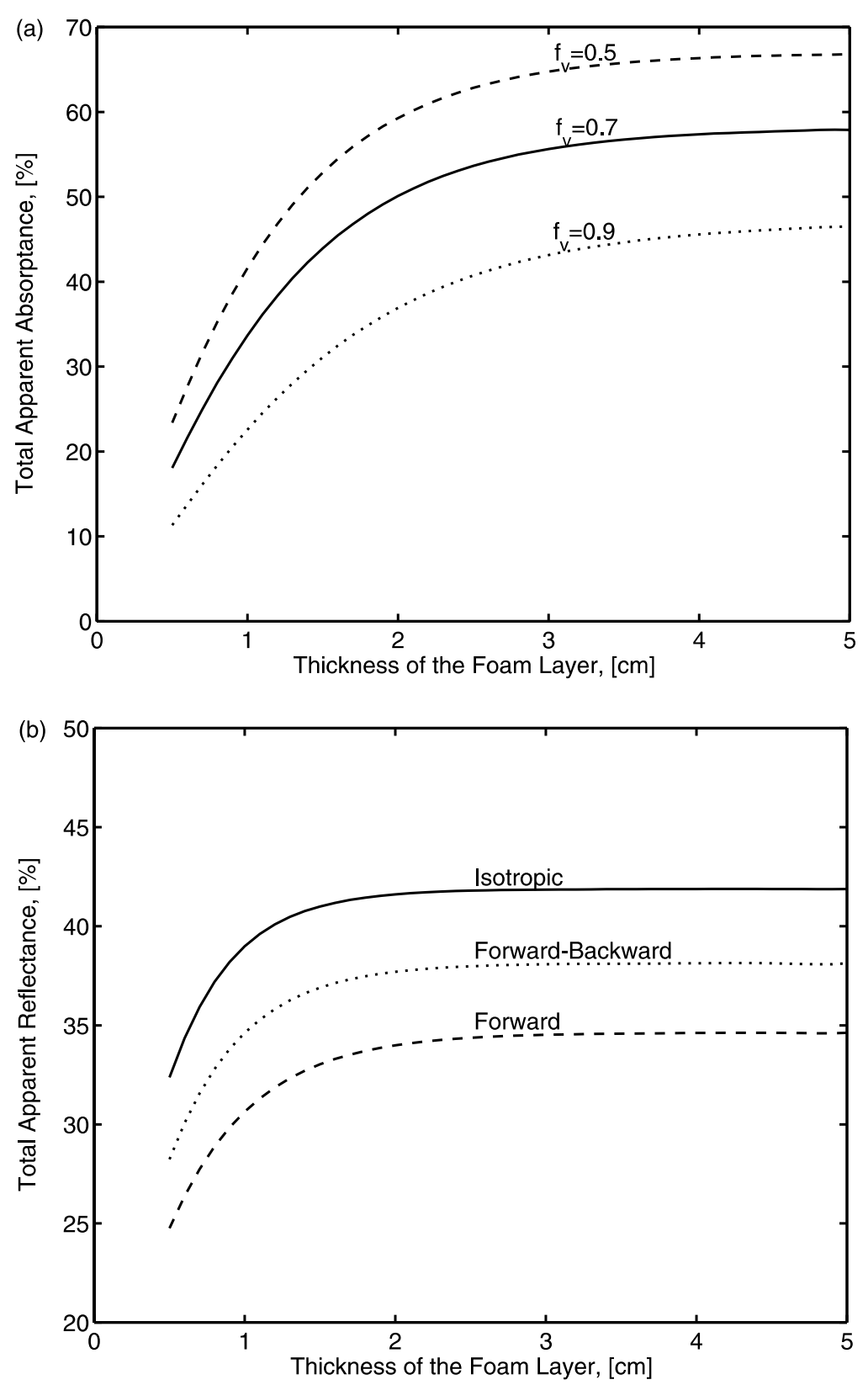

Fig. 8. (a) Effect of the bubble shape on the absorptance $A$ of the foam layer (volume fraction $f_{\mathrm{v}}=0.5$ corresponds to a foam consisting of spherical bubbles only; $f_{\mathrm{v}}=0.9$ corresponds to a foam consisting of polyhedral bubbles only; and $f_{\mathrm{v}}=0.7$ corresponds to a foam consisting of 50\%: 50\% mixture of spherical and polyhedral bubbles); (b) effect of the scattering phase function (i.e., preferred scattering direction of incident radiation by the foam) on the reflectance $R$ of thermal radiation by the glass foam layer.

semitransparent shell, which is a simple yet realistic representation of the unit cell of the primary foam formed on top of batch logs (see Fig. 2(a)). In the case of the large bubble and thick lamella (as compared to the wavelength of the incident radiation), the numerical ray tracing technique can be successfully used to predict the absorption and scattering cross-sections and the 
scattering phase function of the foams. Because the incident radiation can be assumed azimuthally isotropic, the unit cell needs to be discretized only in a two-dimensional axisymmetric plane and the radiation intensity field is computed within a twodimensional plane only. The incident radiation $\left(I_{0}\right)$ is broken into discrete energy bundles of the normalized uniform intensity. In the problem considered, the bundle trajectory is purely deterministic and, thus, a simple tracing of the energy bundles, as they travel within the lamella, leads to the same result as a probabilistic Monte Carlo approach but without additional computations. Each energy bundle is traced within the lamella using the reflection parameters (i.e., an angle and the reflectivity) computed using Fresnel's equations until the bundle is absorbed by the substrate (batch) or its energy is completely attenuated within the lamella.

The ray tracing algorithm described has been validated in the case of a thin hemispherical shell by comparing with an analytical solution of the problem, and it was shown to be very robust and capable of producing reliable results [31]. The results of calculations shown in Fig. 9(a) [32] indicate that the total apparent reflectance and absorptance of the batch foam can be as large as $15 \%$ and $10 \%$, respectively, thereby decreasing the radiation transmission and batch heating rate to only about $75 \%$ of the total incident radiant energy or even lower. Furthermore, Fig. 9(b) shows that the scattering phase function of the primary foams is very well approximated by the commonly used Henyey-Greenstein (HG) phase function [42, p. 416].

\subsection{Combined conduction and radiation heat trans- fer in glass foams}

The analysis of radiative heat transfer in glass and batch foams discussed in the previous section

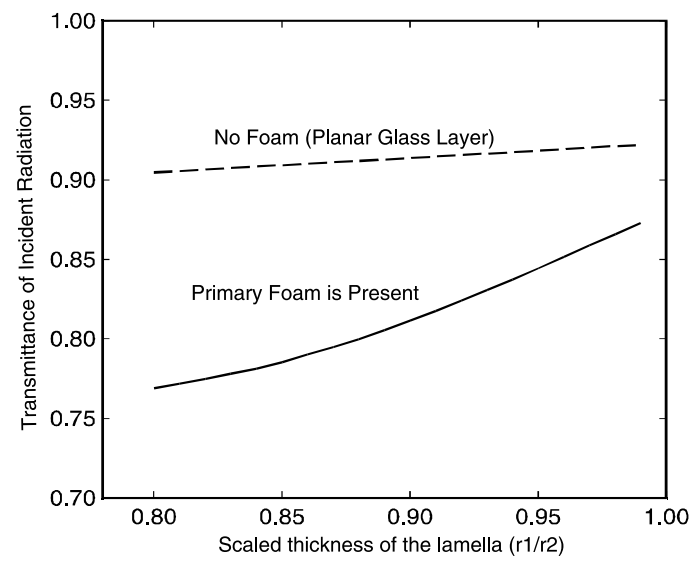

(a)

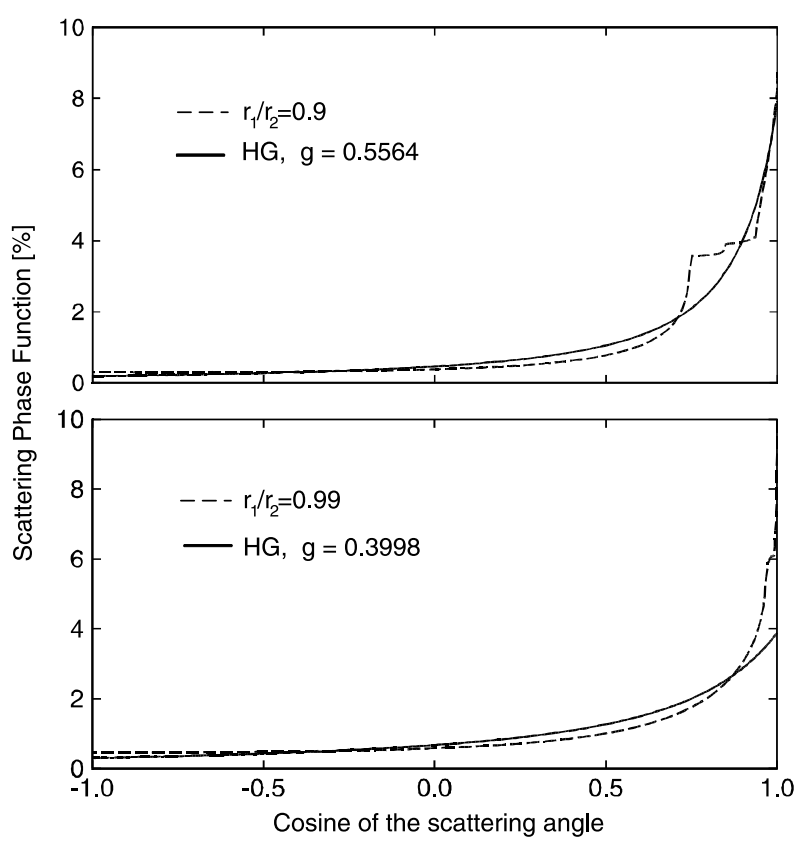

(b)

Fig. 9. (a) Comparison of the transmittance of radiation to the batch through a thin planar layer of molten glass on the top of the batch (no foam case) and in the case when the batch foam is present (modeled as a thin hemispherical shell) as a function of the scaled lamella thickness $\left(r_{1} / r_{2}\right)$ (note that the greater is the radius ratio $r_{1} / r_{2}$ of the inner to the outer hemispheres defining the foam unit cell, the thinner is the foam lamella); (b) comparison of the scattering phase function (i.e., preferred direction of for scattering of incident radiation) of the batch foam with the HG approximation as a function of the cosine of the scattering angle for different scaled foam lamella thicknesses $\left(r_{1} / r_{2}\right)$. 
was instrumental in gaining a critical insight into the problem, however, a number of assumptions has been made in the analysis and also several important features/phenomena unique to glass foams have been intentionally neglected to simplify the problem formulation, thereby making it amenable to purely analytical solution. Specifically: the foam layer was assumed to be isothermal (i.e., radiation was taken to be the only mode of heat transfer considered) and 'cold' (i.e., selfemission of radiation was ignored) medium as compared to the combustion gases in the furnace; the bubbles in the foam were considered motionless; the bubble size distribution function has been assumed non-uniform but stationary across the thickness of the foam layer; the shape of the bubbles (spherical at the bottom and polyhedral at the top of the layer) has been accounted for only implicitly through the effective void fraction variation without explicitly incorporating the bubble shape effects into the behavior of the scattering phase function; the dependent scattering effects have been neglected and the characteristic dimensions of all scatterers were taken to be much larger than the wavelength of the incident IR radiation; the absorption and scattering cross-sections have been calculated based on the closed-form albeit simplified analytical expressions resulted from the Mie theory in the limit of the weakly absorbing media.

Although our scaling analysis [19] showed that many of these assumptions are reasonably accurate as the first approximation, the extent of their validity required more rigorous analysis. More importantly, despite the dominant role of thermal radiation in energy transport from the combustion space to the glass melt, neglecting heat conduction (due to significant temperature gradient across the foam layer) and self-emission of thermal radiation by the foam as well as their intimate coupling and interaction with radiation heat transfer within the foam can hardly be justified. To elucidate the importance of these effects, we have performed an analysis of combined conduction and radiation in glass foams [33].

Fig. 10(a) shows the temperature distribution across the foam layer with the characteristics typically found in glass melting furnaces (called a 'baseline' case) as compared to the idealized foam layer with the zero resistance to radiative heat transfer. Clearly, attenuation of thermal radiation by the foam layer results in a significant decrease of the rate of heat transfer from the combustion space to the glass melt and, in turn, in strong reduction of the glass surface temperature (by as much as $500{ }^{\circ} \mathrm{C}$ in the case of the $5 \mathrm{~cm}$ thick foam layer). An increase in the thickness of the foam layer, as shown in Fig. 10(b), not only leads to a significant decrease in both the heat transfer rate and the surface temperature of the glass melt, but also results in an increased temperature of the top surface of the foam and, thus, in an increased amount of thermally actuated $\mathrm{NO}_{x}$ pollution. These facts underscore the critical importance of understanding heat transfer in foams to improvement of the glass melting process as well as to mitigation of its environmental implications.

\subsection{Practical solutions for reducing foaming}

Given the negative effects of the foam presence on the energy efficiency, glass quality, furnace lifetime, and productivity, practical solutions to reduce foaming in glass melting furnaces are briefly discussed next. In general, batch expansion or primary foaming can be reduced by inducing the release of gases before the first liquid phase appears and becomes interconnected or by accelerating the release of gases to the combustion space [12]. In particular, experimental observations suggest the following practical measures for limiting primary foaming:

1. Slow heating of fine silicate grains favors the release of gases before the liquid phase is formed, thereby reducing primary foaming [12].

2. Rapid heating of coarse silicate grains shifts the equilibrium of batch reactions towards higher temperatures at which the viscosity of the liquid phase is significantly reduced, thereby easing the escape of gases to the atmosphere [12].

3. Addition of $\mathrm{Na}_{2} \mathrm{SO}_{4}$ to the batch limits retention of gases within the glass melt [1,12]. Kim and Hrma [10,12,43] suggested several possible physical mechanisms and theoretical models explaining such an effect, albeit recognizing that 

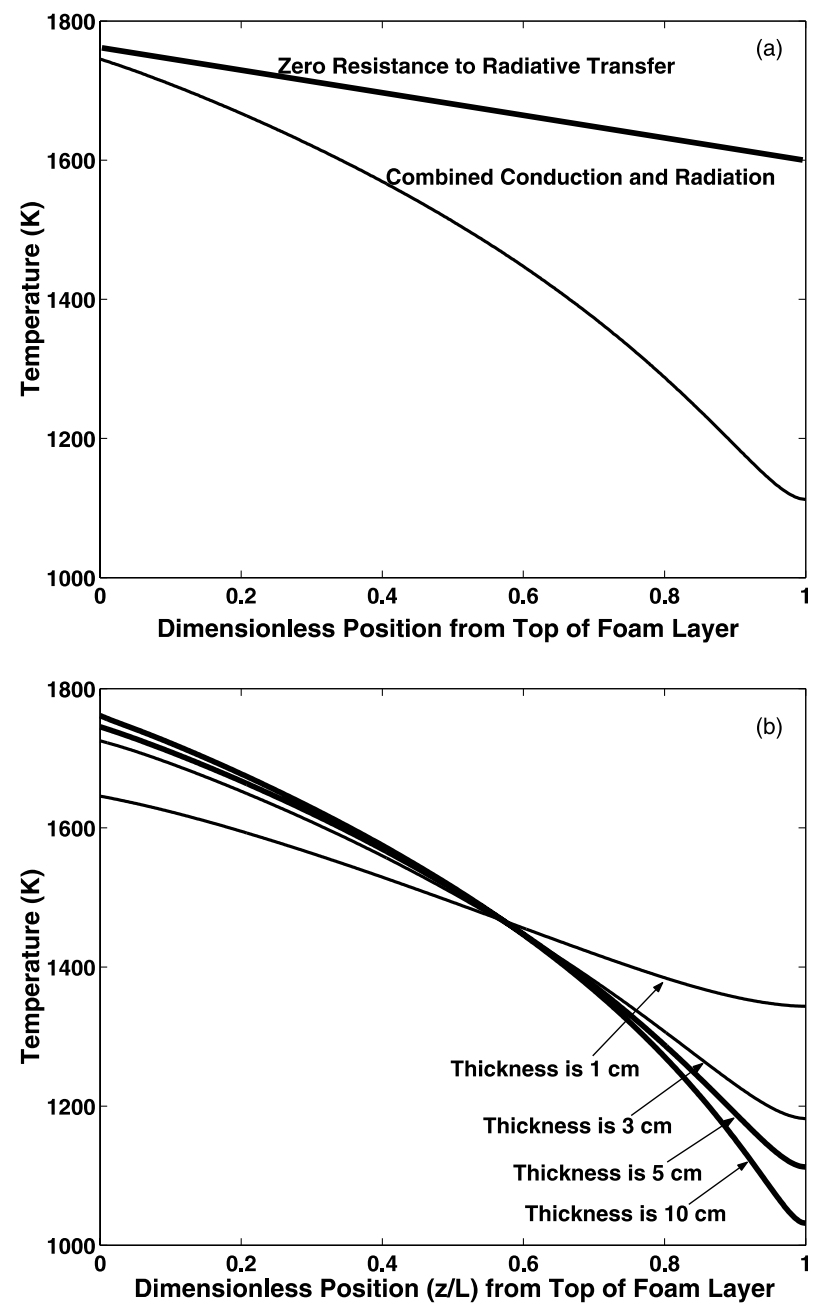

Fig. 10. Temperature distribution across the foam layer: (a) comparison of a typical case for combined conduction and radiation heat transfer against an idealized foam layer with zero resistance to radiative heat transfer for the $5 \mathrm{~cm}$ thick foam layer; (b) effect of the foam thickness on heat transfer in glass foams.

none of them is entirely satisfactory. Moreover, one should note that although $\mathrm{Na}_{2} \mathrm{SO}_{4}$ reduces primary foaming, it also increases secondary foaming by enhancing refining reactions in the glass melt. Therefore, the amount of $\mathrm{Na}_{2} \mathrm{SO}_{4}$ added to the batch needs to be optimized by considering both primary and secondary foam formation.

4. A decrease in the pressure of the surrounding atmosphere reduces the mass of gas retained in the batch, thereby limiting primary foam formation. However, it also lowers the tempera- ture at which carbon dioxide is generated in the batch and thus promotes undesirable batch expansion [12].

5. The size of the batch particles plays an important role in primary foam formation $[7,10,43]$. The use of finer grains accelerates melting and also results in higher temperatures at the top of the batch, thereby sealing the top part of the batch and preventing gases generated at the bottom from escaping to the atmosphere [12]. Thus, an increase in the particle size of the batch powder and of the recycled cullet 
results in reduction of primary foaming [1]. Ahn and Hrma [8] also showed that compacting and heat treating of the simulated nuclear waste batch above $1150{ }^{\circ} \mathrm{C}$ prior to its introduction into the glass melting furnace limits significantly the extent of the primary foam. Finally, retention of gases generated by batch melting and, in turn, primary foam formation are also reduced by adding melting agents such as $\mathrm{Na}_{2} \mathrm{NO}_{3}$ to the batch and by decreasing the amount of $\mathrm{SrCO}_{3}$ and $\mathrm{Al}_{2} \mathrm{O}_{3}$ in the batch [12].

Various means for reducing secondary foaming have been studied extensively, particularly when sodium sulfate $\mathrm{Na}_{2} \mathrm{SO}_{4}$ is used as a refining agent $[1,11,13,39]$. The main findings are the following:

1. An increase in the furnace atmosphere/flame temperature tends to destabilize the foam $[7,9]$ with an exponential, Arrhenius type dependence of the foam decay rate on the temperature [9]. The authors speculate that this effect is due to the exponential decrease of the glass melt viscosity as the temperature increases.

2. Kappel et al. [9] observed that an increase in the pressure and pressure fluctuations of the furnace atmosphere favors decay of the foam, thereby suggesting the use of ultrasonic waves to destroy the foam [44].

3. An increase in the $\mathrm{SO}_{2}$ concentration in the foam bubbles (e.g., provided by the refining reactions) causes the glass foam to decay faster [9].

4. Introduction of pulses of reducing gases (e.g., $\mathrm{CO})$ to the furnace atmosphere was found to be an effective way to destroy secondary foams $[1,11]$. Thus, as a practical solution, Laimbock [1] suggested a sudden change from oxidizing to reducing firing in the combustion space. This method, however, has a number of shortcomings, both environmental and economic (e.g., reduced lifetime of refractories) [1].

5. Spraying of oil, natural gas, or any combustible material as well as $\mathrm{Na}_{2} \mathrm{SO}_{4}, \mathrm{NaOH}$, and $\mathrm{KOH}$ solutions on top of the secondary foam blanket was also found to be an efficient way of the foam destruction [1].
6. In furnaces equipped with oxy-fuel burners, the amount of refining agents added to the batch needs to be optimized. Laimbock [1] proposed a semi-empirical expression for the optimal amount of sodium sulfate that should be added to the batch in production of soda-lime silicate glasses. It accounts for the maximum melting temperature, the water vapor partial pressure in the combustion space, the batch weight, the sulfate losses during initial fusion/melting reactions, and the small variations in the batch composition [1].

7. Addition of active carbon (e.g., coke) to the batch reduces secondary foaming occurring in the high temperature regions of the furnace. However, it also promotes formation of primary foams [11].

It should be noted that all previously discussed experimental results were obtained in the laboratory crucibles, thus, one should be careful in extending these results to actual glass melting furnaces [12]. Some of the more important effects that are difficult to observe in the small scale laboratory systems are as follows: (i) the refining reactions observed in crucibles at high temperatures would most probably occur only at the top of the batch in the actual furnace that is directly exposed to radiant heating, whereas the bottom of the batch is too cold for initiation of fusion reactions due to the 'insulation effect' of the expanded batch; (ii) partitioning of the gases generated in the batch between what is released (escaped) to the combustion space and what is entrapped in the form of gas bubbles in the glass melt and then carried away by the convection currents is difficult if not impossible to study in laboratory systems.

\section{Concluding remarks}

This review presents the current state-of-the-art in understanding and modeling of glass foams including mechanisms of formation, foam stability, transport properties, and heat, mass, and radiation transfer. Evidently, there has been some progress made over the years and especially recently in this area, however, there is still a number of very im- 
portant issues that require further research and some of them are:

- Understanding and modeling of the processes defining formation of the primary and secondary foams including the retention of gas bubbles generated within the batch and their partitioning between the release to the combustion space and entrapment within the glass melt. Such a model should account for the effects of the batch grain size, the fusion/melting reactions and $\mathrm{CO}_{2}$ generation, entrapment and escape of gases generated in the batch, the refining reactions, the change of the effective thermal conductivity of the batch due to the presence of entrapped gases, and the temperature gradients in the batch.

- The effects of the bubble size distribution, the conditions of the furnace atmosphere (i.e., chemical composition, pressure, temperature and their fluctuations), liquid phase evaporation, and the bubbling gas solubility and diffusivity in the glass melt need to be explored and quantified in predicting the transient and steady-state thickness of the foam layer.

- Experiment data and mathematical models for rheological properties of the secondary foams and their dependence on the foam structure, chemical composition, and thermophysical properties are needed for predicting the spreading of the foam over the glass melt surface.

- Development of quantitative models to predict the minimum superficial gas velocity required for onset of foaming.

- Combined radiation, conduction, and convection in high temperature batch and glass foams to account for: (1) emission, absorption, and scattering of thermal radiation, (2) heat conduction due to large temperature gradient across the foam layer, (3) heat convection due to collective up-rising bubble motion and intrabubble gas circulation, (4) coupling and interaction effects.

- Effective radiative properties of batch and glass foams: (1) compare the detailed electromagnetic calculations with the results of the simplified model [19] for different sizes of bubbles and thicknesses of the lamellae; (2) develop an ap- proach for calculating the scattering phase function for polyhedral bubbles; (3) explicitly account for change in the bubble size and shape distribution functions across the foam thickness; (4) account for the effect of foam stabilizing surfactants on the effective radiative properties of the foam; (5) account for the cross-correlation effects on radiative properties due to collective up-rising motion of bubbles.

- Revisit the question of dependent vs. independent scattering by glass foams, considering the fact the thickness of the foam lamella could be of the same order of magnitude as the wavelength of incident radiation and develop the models for accounting for near-field and multiple scale scattering effects.

- Develop a consistent set of experimental data to verify the predictions of the theoretical models.

In conclusion, we would like to emphasize a tremendous practical importance of the fundamental understanding and experimentally verified mathematical modeling of glass foams that, combined with comprehensive modeling of the combustion space and glass melt in the glass melting furnace, will provide a foundation for development of the energy efficient and environmentally conscious technology for manufacturing of high quality glass products.

\section{Acknowledgements}

The authors are indebted to the US Department of Energy and to Argonne National Laboratory for their financial support within the framework of the US Department of Energy/Glass Industry/Argonne National Laboratory/University collaborative research project.

\section{References}

[1] P.R. Laimbock, PhD thesis, Technical University of Eindhoven, 1998.

[2] R.G.C. Beerkens, Glastech. Ber. 71 (12) (1995) 369.

[3] S. Kawachi, Y. Kawase, Glastech. Ber. 71 (4) (1998) 83.

[4] S. Kawachi, Y. Kawase, Glastech. Ber. 71 (5) (1998) 111.

[5] R. Viskanta, J. Non-Cryst. Solids 177 (1994) 347.

[6] W. Trier, Glastech. Ber. 36 (3) (1963) 73-86. 
[7] A. Gerrard, I.H. Smith, Laboratory techniques for studying foam formation and stability in glass melting, Glastechnische Berichte-XIII. Internationaler Glaskongress, Hamburg, vol. 56K, 4-9 July 1983, p. 13.

[8] J.S. Ahn, P. Hrma, Advances in Ceramics, Nuclear Waste Management II, vol. 20, 1986, p. 181.

[9] J. Kappel, R. Conradt, H. Scholze, Glastech. Ber. 60 (6) (1987) 189.

[10] D.-S. Kim, P. Hrma, J. Am. Ceram. Soc. 74 (3) (1991) 551.

[11] R.G.C. Beerkens, P. Laimbock, in: J. Kieffer (Ed.), 60th Conference on Glass Problems-Urbana, IL, American Ceramic Society, October 19-20, 1999, p. 41.

[12] D.-S. Kim, P. Hrma, J. Am. Ceram. Soc. 69 (6) (1990) 1039.

[13] R.G.C. Beerkens, L. Zaman, P. Laimbock, S. Kobayashi, Glastech. Ber. 72 (5) (1999) 127.

[14] R.G.C. Beerkens, H.D. Wall, J. Am. Ceram. Soc. 73 (7) (1990) 1857.

[15] E. Itoh, H. Yoshikawa, H. Miura, Y. Kawase, Glass Technol. 38 (4) (1997) 134.

[16] S. Kawachi, M. Kato, Glastech. Ber. 72 (6) (1999) 182.

[17] M. Cable, D. Martlew, Glass Technol. 37 (4) (1996) 137.

[18] L. Nemec, Glass Technol. 21 (3) (1980) 134.

[19] A.G. Fedorov, R. Viskanta, Phys. Chem. Glasses 41 (3) (2000) 127.

[20] A.G. Fedorov, R. Viskanta, J. Am. Ceram. Soc. 83 (11) (2000) 2769.

[21] G. Narsimhan, E. Ruckenstein, Lanmuir 2 (1986) 230.

[22] A. Bhakta, E. Ruckenstein, Adv. Colloid Interf. Sci. 70 (1997) 1.

[23] Y. Zhang, R.J. Fruehan, Metall. Mater. Trans. B 26B (1995) 803.

[24] B. Horan, Foam samples from the techneglas columbus $h$ furnace, personal communication, 14th June, 2001.

[25] P. Hrma, J. Colloid Interf. Sci. 134 (1) (1990) 161.

[26] R. Viskanta, M.P. Menguc, Appl. Mech. Rev. 42 (1989) 241.

[27] R. Viskanta, X. Wu, Glastech. Ber. 56 (1983) 138.

[28] L. Pilon, A.G. Fedorov, R. Viskanta, J. Cell. Plast. 36 (2000) 451.
[29] L. Pilon, A.G. Fedorov, R. Viskanta, J. Colloid Interf. Sci. 242 (2) (2001) 425.

[30] L. Pilon, A.G. Fedorov, R. Viskanta, Chem. Eng. Sci. 57 (2002) 977.

[31] T.-H. Fan, A.G. Fedorov, J. Quant. Spectrosc. Radiat. Transfer 73 (2002) 285; Proceedings of the 3rd International Symposium on Radiative Transfer, ICHMT, Antalya, Turkey, June 17-22, 2001.

[32] T.-H. Fan, A.G. Fedorov, ASME J. Heat Transfer, in press, in: Proceedings of International Mechanical Engineering Congress \& Exposition IMECE'01, ASME, New York City, NY, November 2001.

[33] M. Varady, A.G. Fedorov, ASME J. Heat Transfer, in review, Proceedings International Mechanical Engineering Congress \& Exposition IMECE'01, ASME, New York City, NY, November 2001.

[34] A.G. Fedorov, Proceedings of the 5th ISHMT/ASME Heat and Mass Transfer Conference, Calcutta, India, January 2002.

[35] S. Hartland, A.D. Barber, Trans. Institut. Chem. Eng. 52 (1974) 43.

[36] S.A.K. Jeelani, S. Ramaswami, S. Hartland, Trans. Institut. Chem. Eng., Part A 68 (1990) 271.

[37] C.F. Cooper, J.A. Kitchener, J. Iron Steel Inst. (September) (1959) 48.

[38] M. Cable, C.G. Rasul, J. Savage, Glass Technol. 9 (2) (1968).

[39] R.G.C. Beerkens, P. Laimbock, Ceram. Eng. Sci. Proc. 21 (1) $(2000) 41$.

[40] N.M. Parikh, J. Am. Ceram. Soc. 41 (1958) 18.

[41] Geotti-Bianchini, J.T. Brown, A.J. Faber, H. Hessenkemper, S. Kobayashi, I.H. Smith, Glastech. Ber. 72 (5) (1999) 145.

[42] M.F. Modest, Radiative Heat Transfer, McGraw-Hill, New York, NY, 1993.

[43] D.-S. Kim, P. Hrma, J. Am. Ceram. Soc. 75 (11) (1992) 2959.

[44] S.V. Komarov, M. Kuwabara, M. Sano, ISIJ Int. 39 (12) (1999) 1207. 